\title{
SOME QUASI-STATIC PROBLEMS WITH ELASTIC AND VISCOUS BOUNDARY CONDITIONS IN LINEAR VISCOELASTICITY
}

\author{
BY
}

\author{
CARLO ALBERTO BOSELLO AND GIORGIO GENTILI
}

Dipartimento di Matematica, Università degli Studi di Bologna, Italy

\begin{abstract}
We study the quasi-static behaviour of a linearly viscoelastic body which is subject to boundary forces respectively of elastic type and of viscous type. The ensuing problems exhibit dynamic boundary conditions. We impose on the memory kernel only those restrictions deriving from thermodynamics and, making use of the Fourier transform method, we show existence and uniqueness of the solution to each problem.
\end{abstract}

1. Introduction. A linearly viscoelastic body is described by the constitutive equation:

$$
\mathbf{T}(\mathbf{x}, t)=G_{0}(\mathbf{x}) \mathbf{E}(\mathbf{x}, t)+\int_{0}^{\infty} \dot{G}(\mathbf{x}, s) \mathbf{E}(\mathbf{x}, t-s) d s
$$

where $\mathbf{T}$ is the Cauchy stress (second-order) tensor, $\mathbf{E}=\frac{1}{2}\left(\nabla \mathbf{u}+\nabla \mathbf{u}^{\mathrm{T}}\right), \mathbf{u}$ is the displacement vector, and

$$
G(\mathbf{x}, t)=G_{0}(\mathbf{x})+\int_{0}^{t} \dot{G}(\mathbf{x}, s) d s, \quad t>0
$$

is a symmetric fourth-order tensor representing the relaxation function of the viscoelastic material. The quasi-static behaviour of a continuum medium is described by the equation

$$
\nabla \cdot \mathbf{T}(\mathbf{x}, t)+\mathbf{f}(\mathbf{x}, t)=\mathbf{0}, \quad(\mathbf{x}, t) \in \Omega \times \mathbb{R}
$$

together with suitable boundary conditions (here $\Omega$ is an open and bounded region of $\mathbb{R}^{3}$ with sufficiently regular boundary).

For materials of type (1.1), (1.3) turns out to be an integro-differential equation of elliptic type, depending on time, whose integral kernel is $\dot{G}$. We shall assume that $G$ satisfies the fading memory principle, at least in its weak form (see for instance [1]). Furthermore, we shall impose on $G$ the restriction dictated by the Second Law of Thermodynamics in the Clausius form ([7]). In particular, as Fabrizio and Morro pointed out ([3] and [4]), we shall distinguish reversible from irreversible processes in the sense that,

Received May 25, 1994.

1991 Mathematics Subject Classification. Primary 73F99, 45K05, 35J25.

(C)1996 Brown University 
in the Second Law, equality holds for reversible processes only. Such restrictions on $G$ are summarized in the following properties:

i) $\dot{G}(\mathbf{x}, \cdot) \in L^{1}\left(\mathbb{R}^{+}\right) \quad \forall \mathbf{x} \in \bar{\Omega} ; \quad G(\cdot, t), \dot{G}(\cdot, t) \in C^{1}(\bar{\Omega}) \quad \forall t \geq 0$

ii) $G(\mathbf{x}, t)$ is a symmetric tensor in $\bar{\Omega} \times \mathbb{R}^{+}$; moreover, $G_{0}(\mathbf{x})$ and $G_{\infty}(\mathbf{x})=$ $\lim _{t \rightarrow \infty} G(\mathbf{x}, t)$ are positive definite, that is, there exist two positive constants $\gamma_{0}$ and $\gamma_{\infty}$ such that

$$
G_{0}(\mathbf{x}) \mathbf{E} \cdot \mathbf{E} \geq \gamma_{0}|\mathbf{E}|^{2}, \quad G_{\infty}(\mathbf{x}) \mathbf{E} \cdot \mathbf{E} \geq \gamma_{\infty}|\mathbf{E}|^{2} \quad \forall \mathbf{x} \in \bar{\Omega} ;
$$

iii) the "sine" Fourier transform of $\dot{G}: \hat{\dot{G}}_{s}(\mathbf{x}, \omega)=\int_{0}^{\infty} \dot{G}(\mathbf{x}, s) \sin \omega s d s$ is such that

$$
\hat{\dot{G}}_{s}(\mathbf{x}, \omega) \mathbf{E} \cdot \mathbf{E}<0 \quad \forall \mathbf{x} \in \bar{\Omega}, \forall \omega>0, \forall \mathbf{E} \neq \mathbf{0} .
$$

REMARK. Indeed, the condition that $G_{\infty}$ be positive definite does not really follow from the above assumptions but rather from experimental evidence. Such a property is specific for viscoelastic solids (see [10]).

A quasi-static problem for a viscoelastic body has recently been studied in [2] and [5]; it had homogeneous Dirichlet boundary conditions, namely,

$$
\begin{cases}\nabla \cdot \mathbf{T}(\mathbf{x}, t)+\mathbf{f}(\mathbf{x}, t)=\mathbf{0} & (\mathbf{x}, t) \in \Omega \times \mathbb{R} \\ \mathbf{u}(\mathbf{x}, t)=\mathbf{0} & (\mathbf{x}, t) \in \partial \Omega \times \mathbb{R}\end{cases}
$$

It has been shown that restrictions i)-iii) are necessary and sufficient for the wellposedness of the problem (1.6), that is, to guarantee existence and uniqueness of solutions.

In this work we assume less simple boundary conditions. They are of dynamic type and are to some extent related respectively to an elastic and a viscous force acting on the boundary. We apply the Fourier transform method and show that, if the relaxation function $G$ satisfies restrictions i)-iii), the quasi-static problem with dynamic boundary conditions still admits one and only one solution.

In Section 2 we consider a boundary condition of the form $\sigma(\mathbf{x}) \mathbf{u}(\mathbf{x}, t)+\mathbf{T}(\mathbf{x}, t) \cdot \mathbf{n}=\mathbf{0}$, where $\sigma(\mathbf{x})>0$ represents the elastic constant of the boundary force, whereas in Section 3 we consider mixed boundary conditions: we fix $\mathbf{u}=\mathbf{0}$ on $\Gamma_{0} \subset \partial \Omega$ and assume $\lambda(\mathbf{x}) \dot{\mathbf{u}}(\mathbf{x}, t)+\mathbf{T}(\mathbf{x}, t) \cdot \mathbf{n}=\mathbf{0}$ on $\Gamma_{1}=\partial \Omega \backslash \Gamma_{0}$, where $\lambda(\mathbf{x})>0$ is the viscosity coefficient. Finally, the case when $\Gamma_{0}=\varnothing$ is discussed. The latter is especially interesting since, in general, such a problem cannot be solved and, in particular, nonuniqueness of solutions arises (as in the similar problem with Neumann boundary conditions); nevertheless, the viscosity at the boundary guarantees uniqueness of the solution among functions that are $L^{2}$ in time and also ensures its existence provided the volume force and its impulse are $L^{2}$ in time.

2. Elastic constraint on the boundary. Let us consider the quasi-static problem of a viscoelastic body of the type (1.1) occupying a bounded region $\Omega$ ( $\Omega$ is an open connected subset of $\mathbb{R}^{3}$ ) and subject to an elastic force on the boundary $\partial \Omega$ (which is supposed to be sufficiently regular). Such a problem has the form

$$
\begin{cases}\nabla \cdot \mathbf{T}(\mathbf{x}, t)+\mathbf{f}(\mathbf{x}, t)=\mathbf{0} & (\mathbf{x}, t) \in \Omega \in \mathbb{R} \\ \sigma(\mathbf{x}) \mathbf{u}(\mathbf{x}, t)+\mathbf{T}(\mathbf{x}, t) \cdot \mathbf{n}=\mathbf{0} & (\mathbf{x}, t) \in \partial \Omega \times \mathbb{R}\end{cases}
$$


where $\mathbf{n}$ is the outward normal to $\partial \Omega$ and $\sigma(\mathbf{x})>0 \forall \mathbf{x} \in \partial \Omega$ is the elastic constant relative to the force on $\partial \Omega$. Here the volume force $\mathbf{f} \in L^{2}\left(\mathbb{R}, L^{2}(\Omega)\right)$ plays the role of the supply. Substituting (1.1) into (2.1) we obtain the integro-differential problem

$$
\begin{cases}\nabla \cdot\left(G_{0}(\mathbf{x}) \nabla \mathbf{u}(\mathbf{x}, t)+\int_{0}^{\infty} \dot{G}(\mathbf{x}, s) \nabla \mathbf{u}(\mathbf{x}, t-s) d s\right)+\mathbf{f}(\mathbf{x}, t)=\mathbf{0} & (\mathbf{x}, t) \in \Omega \times \mathbb{R}, \\ \sigma(\mathbf{x}) \mathbf{u}(\mathbf{x}, t)+G_{0}(\mathbf{x}) \nabla \mathbf{u}(\mathbf{x}, t) \cdot \mathbf{n}+\int_{0}^{\infty} \dot{G}(\mathbf{x}, s) \nabla \mathbf{u}(\mathbf{x}, t-s) \cdot \mathbf{n} d s=\mathbf{0} & (\mathbf{x}, t) \in \partial \Omega \times \mathbb{R} .\end{cases}
$$

We first seek weak solutions for the integro-differential problem (2.2). They and their functional spaces as $\mathbf{f} \in L^{2}\left(\mathbb{R}, L^{2}(\Omega)\right)$ will be determined through the following definition (see [11], chap. III, Theorem 3.A):

DEFInItion 2.1. A function $\mathbf{u}$ is said to be a weak solution of the integro-differential problem (2.2) with $\mathbf{f} \in L^{2}\left(\mathbb{R}, L^{2}(\Omega)\right)$ if $\mathbf{u} \in L^{2}\left(\mathbb{R}, H^{1}(\Omega)\right)$ and if the problem in variational form

$$
\begin{aligned}
\int_{\Omega}\left(G_{0}(\mathbf{x}) \nabla \mathbf{u}(\mathbf{x}, t)+\int_{0}^{\infty} \dot{G}(\mathbf{x}, s) \nabla \mathbf{u}(\mathbf{x},\right. & t-s) d s) \cdot \nabla \mathbf{v}(\mathbf{x}) d \mathbf{x} \\
& +\int_{\partial \Omega} \sigma(\mathbf{x}) \mathbf{u}(\mathbf{x}, t) \mathbf{v}(\mathbf{x}) d \mathbf{a}=\int_{\Omega} \mathbf{f}(\mathbf{x}, t) \mathbf{v}(\mathbf{x}) d \mathbf{x}
\end{aligned}
$$

is satisfied $\forall \mathbf{v} \in H^{1}(\Omega)$ and $\forall t \in \mathbb{R}$.

In order to prove existence and uniqueness of solutions we consider the Fourier transform of (2.2). From now on, we identify $\dot{G}(\mathbf{x}, \cdot)$ with its causal extension to $\mathbb{R}$, that is, we consider $\dot{G}(\mathbf{x}, s)=0$ when $s<0$, so that the Fourier transform $\hat{\dot{G}}$ of $\dot{G}$ can be related to its "sine" $\hat{\dot{G}}_{s}$ and "cosine" $\hat{\dot{G}}_{c}$ Fourier transforms as follows:

$$
\hat{\dot{G}}(\mathbf{x}, \omega)=\hat{\dot{G}}_{c}(\mathbf{x}, \omega)-i \hat{\dot{G}}_{s}(\mathbf{x}, \omega) .
$$

Therefore, if $\mathbb{G}$ denotes the following fourth-order symmetric tensor:

$$
\mathbb{G}(\mathbf{x}, \omega)=G_{0}(\mathbf{x})+\hat{\dot{G}}(\mathbf{x}, \omega),
$$

the transformed problem of (2.2) is defined for each $\omega \in \mathbb{R}$ as

$$
\begin{cases}\nabla \cdot(\mathbb{G}(\mathbf{x}, \omega) \nabla \hat{\mathbf{u}}(\mathbf{x}, \omega))+\hat{\mathbf{f}}(\mathbf{x}, \omega)=\mathbf{0} & \mathbf{x} \in \Omega \\ \sigma(\mathbf{x}) \hat{\mathbf{u}}(\mathbf{x}, \omega)+(\mathbb{G}(\mathbf{x}, \omega) \nabla \hat{\mathbf{u}}(\mathbf{x}, \omega)) \cdot \mathbf{n}=\mathbf{0} & x \in \partial \Omega .\end{cases}
$$

In this way, we reduce the study of an integro-differential problem to a family of elliptic problems depending on a parameter $\omega \in \mathbb{R}$.

Definition 2.2. For each fixed $\omega, \hat{\mathbf{u}}$ is said to be a weak solution of problem (2.5) if $\hat{\mathbf{u}} \in H^{1}(\Omega)$ and for each $\mathbf{v} \in H^{1}(\Omega)$ we have

$$
\int_{\Omega} \mathbb{G}(\mathbf{x}, \omega) \nabla \hat{\mathbf{u}}(\mathbf{x}) \nabla \mathbf{v}^{*}(\mathbf{x}) d \mathbf{x}+\int_{\partial \Omega} \sigma(\mathbf{x}) \hat{\mathbf{u}}(\mathbf{x}) \mathbf{v}^{*}(\mathbf{x}) d \mathbf{a}=\int_{\Omega} \hat{\mathbf{f}}(\mathbf{x}, \omega) \mathbf{v}^{*}(\mathbf{x}) d \mathbf{x} .
$$

REMARK 2.1. Observe that the Fourier transform and its inverse are continuous mappings of $L^{2}$ onto itself. Therefore, if $\mathbf{u}$ is a weak solution of $(2.2)$ then $\hat{\mathbf{u}}(\cdot, \omega)$ is a weak solution of $(2.5)$ for almost all $\omega \in \mathbb{R}$, whereas if $\hat{\mathbf{u}}(\cdot, \omega)$ is a weak solution of $(2.5)$ for almost all $\omega \in \mathbb{R}$ and $\hat{\mathbf{u}} \in L^{2}\left(\mathbb{R}, H^{1}(\Omega)\right)$, then $\mathbf{u}$ is a weak solution of $(2.2)$. 
Theorem 2.1. If $G$ satisfies i)-iii) then, for each fixed $\omega \in \mathbb{R}$ and for each $\hat{\mathbf{f}}(\cdot, \omega) \in L^{2}(\Omega)$, there exists one and only one weak solution $\hat{\mathbf{u}}(\cdot, \omega) \in H^{1}(\Omega)$ of $(2.5)$.

Proof. For the Lax-Milgram theorem, the thesis is proved if the bilinear form

$$
a(\mathbf{u}, \mathbf{v} ; \omega)=\int_{\Omega} \mathbb{G}(\mathbf{x}, \omega) \nabla \mathbf{u}(\mathbf{x}) \nabla \mathbf{v}^{*}(\mathbf{x}) d \mathbf{x}+\int_{\partial \Omega} \sigma(\mathbf{x}) \mathbf{u}(\mathbf{x}) \mathbf{v}^{*}(\mathbf{x}) d \mathbf{a}
$$

is coercive $([12])$ in $H^{1}(\Omega)$, namely,

$$
|a(\mathbf{u}, \mathbf{u} ; \omega)| \geq C(\omega)\|\mathbf{u}\|_{1, \Omega}^{2} .
$$

(Henceforth $\|\cdot\|_{s, D}$ will denote the norm in the functional space $H^{s}(D)$ and $\|\cdot\|_{, D}=$ $\|\cdot\|_{0, D}$ will denote the norm in $L^{2}(D)$.)

Consider first the case $\omega=0$.

By $(1.4)_{2}$, since $G_{\infty}(\mathbf{x})=G_{0}(\mathbf{x})+\hat{\dot{G}}_{c}(\mathbf{x}, 0)$ and $\sigma=\inf _{\mathbf{x} \in \partial \Omega} \sigma(\mathbf{x})>0$ we obtain

$$
a(\mathbf{u}, \mathbf{u} ; 0) \geq \gamma_{\infty}\|\nabla \mathbf{u}\|_{, \Omega}^{2}+\sigma\|\mathbf{u}\|_{, \partial \Omega}^{2}
$$

When $\omega \neq 0$, since the tensor $\mathbb{G}(\mathbf{x}, \omega)$ is bounded, there exists a positive bounded function $K(\omega)>0$ such that

$$
\left|\mathbb{G}(\mathbf{x}, \omega) \nabla \mathbf{u}(\mathbf{x}) \nabla \mathbf{u}^{*}(\mathbf{x})\right| \leq K(\omega)|\nabla \mathbf{u}(\mathbf{x})|^{2}
$$

Moreover, (1.5) implies that there exists a positive function $\gamma_{s}(\omega)$ such that

$$
-\hat{\dot{G}}_{s}(\mathbf{x}, \omega) \nabla \mathbf{u}(\mathbf{x}) \nabla \mathbf{u}^{*}(\mathbf{x}) \geq \gamma_{s}(\omega)|\nabla \mathbf{u}(\mathbf{x})|^{2} \quad \text { for } \omega>0 \text { if } \nabla \mathbf{u} \neq \mathbf{0}
$$

therefore, we get the following two inequalities:

$$
\begin{gathered}
|a(\mathbf{u}, \mathbf{u} ; \omega)| \geq \operatorname{Re}\{a(\mathbf{u}, \mathbf{u} ; \omega)\} \geq-K(\omega)\|\nabla \mathbf{u}\|_{L^{2}(\Omega)}^{2}+\sigma\|\mathbf{u}\|_{L^{2}(\partial \Omega)}^{2} \\
|a(\mathbf{u}, \mathbf{u} ; \omega)| \geq|\operatorname{Im}\{a(\mathbf{u}, \mathbf{u} ; \omega)\}| \geq \gamma_{s}(\omega)\|\nabla \mathbf{u}\|_{L^{2}(\Omega)}^{2}
\end{gathered}
$$

where $\gamma_{s}(\omega)>0 \forall \omega \neq 0$. After some simple calculations, from (2.9) and (2.10) it follows that

$$
|a(\mathbf{u}, \mathbf{u} ; \omega)| \geq \alpha(\omega)\left[\|\nabla \mathbf{u}\|_{\Omega}^{2}+\|\mathbf{u}\|_{, \partial \Omega}^{2}\right]
$$

where

$$
\alpha(\omega)=\frac{\gamma_{s}(\omega)}{\gamma_{s}(\omega)+2|K(\omega)|} \cdot \min \{|K(\omega)|, \sigma\}>0
$$

It is easy to show (see, for instance, [11], chap. III, Theorem $5 \mathrm{C}$ ) that, if $\mathbf{u} \in H^{1}(\Omega)$ (with $\Omega$ bounded and $\partial \Omega$ sufficiently regular) then its $H^{1}$-norm in $\Omega$ is bounded by the $L^{2}$-norm of $\nabla \mathbf{u}$ in $\Omega$ and the $L^{2}$-norm of the trace of $\mathbf{u}$ in $\partial \Omega$, i.e., there exists a constant $C_{1}(\Omega)>0$ such that

$$
\left[\|\nabla \mathbf{u}\|_{, \Omega}^{2}+\|\mathbf{u}\|_{, \partial \Omega}^{2}\right] \geq C_{1}(\Omega)\|\mathbf{u}\|_{1, \Omega}^{2}
$$

$\forall \mathbf{u} \in H^{1}(\Omega)$. Thus the norm defined by the left-hand side of (2.13) is equivalent to the norm of $H^{1}(\Omega)$. By virtue of (2.11) and (2.13) the coercivity of $a(\mathbf{u}, \mathbf{u} ; \omega)$ is proved for each $\omega \in \mathbb{R}$.

On the basis of the previous result we can state the following theorem. 
Theorem 2.2. If $G$ satisfies i)-iii) then, for every $\mathbf{f} \in L^{2}\left(\mathbb{R}, L^{2}(\Omega)\right)$, the integrodifferential problem (2.2) has one and only one weak solution $\mathbf{u} \in L^{2}\left(\mathbb{R}, H^{1}(\Omega)\right)$.

Proof. By Theorem 2.1, $\forall \omega$ such that $\hat{\mathbf{f}}(\cdot, \omega) \in L^{2}(\Omega)$, problem $(2.5)$ admits one and only one weak solution $\hat{\mathbf{u}}(\cdot, \omega) \in H^{1}(\Omega)$ and we have

$$
|a(\hat{\mathbf{u}}, \hat{\mathbf{u}} ; \omega)|=\left|\int_{\Omega} \hat{\mathbf{f}}(\mathbf{x}, \omega) \hat{\mathbf{u}}^{*}(\mathbf{x}) d \mathbf{x}\right| \leq\|\hat{\mathbf{f}}\|_{, \Omega}\|\hat{\mathbf{u}}\|_{, \Omega} \leq\|\hat{\mathbf{f}}\|_{, \Omega}\|\hat{\mathbf{u}}\|_{1, \Omega} .
$$

By some energy estimates for the transformed problem (2.5), we show now that, if $\hat{\mathbf{f}} \in L^{2}\left(\mathbb{R}, L^{2}(\Omega)\right)$ then $\hat{\mathbf{u}} \in L^{2}\left(\mathbb{R}, H^{1}(\Omega)\right)$. By virtue of (1.4) and of the continuity of $\hat{\dot{G}}(\mathbf{x}, \cdot)$, one can find $\omega_{1}, \omega_{2}>0$ such that

$$
\begin{array}{ll}
{\left[G_{0}(\mathbf{x})+\hat{\dot{G}}_{c}(\mathbf{x}, \omega)\right] \nabla \mathbf{u}(\mathbf{x}) \nabla \mathbf{u}^{*}(\mathbf{x}) \geq \gamma_{1}|\nabla \mathbf{u}(\mathbf{x})|^{2} \quad \forall \omega \in\left[-\omega_{1}, \omega_{1}\right],} \\
{\left[G_{0}(\mathbf{x})+\hat{\dot{G}}_{c}(\mathbf{x}, \omega)\right] \nabla \mathbf{u}(\mathbf{x}) \nabla \mathbf{u}^{*}(\mathbf{x}) \geq \gamma_{2}|\nabla \mathbf{u}(\mathbf{x})|^{2} \quad \forall|\omega| \geq \omega_{2} .}
\end{array}
$$

Inequalities (2.15) and (2.16) imply, respectively,

$$
\begin{aligned}
|a(\hat{\mathbf{u}}, \hat{\mathbf{u}} ; \omega)| \geq \operatorname{Re}\{a(\hat{\mathbf{u}}, \hat{\mathbf{u}} ; \omega)\} & \geq \gamma_{1}\|\nabla \hat{\mathbf{u}}\|_{, \Omega}^{2}+\sigma\|\hat{\mathbf{u}}\|_{, \partial \Omega}^{2} \\
& \geq \alpha_{1}\left[\|\nabla \hat{\mathbf{u}}\|_{, \Omega}^{2}+\|\hat{\mathbf{u}}\|_{, \partial \Omega}^{2}\right] \quad \forall \omega \in\left[-\omega_{1}, \omega_{1}\right], \\
|a(\hat{\mathbf{u}}, \hat{\mathbf{u}} ; \omega)| \geq \operatorname{Re}\{a(\hat{\mathbf{u}}, \hat{\mathbf{u}} ; \omega)\} & \geq \gamma_{2}\|\nabla \hat{\mathbf{u}}\|_{, \Omega}^{2}+\sigma\|\hat{\mathbf{u}}\|_{, \partial \Omega}^{2} \\
& \geq \alpha_{2}\left[\|\nabla \hat{\mathbf{u}}\|_{, \Omega}^{2}+\|\hat{\mathbf{u}}\|_{, \partial \Omega}^{2}\right] \quad \forall|\omega| \geq \omega_{2} .
\end{aligned}
$$

If we denote $I=\left[\omega_{1}, \omega_{2}\right]$ and $\alpha=\min \left\{\alpha_{1}, \alpha_{2}, \inf _{|\omega| \in I} \alpha(\omega)\right\}$, it is easy to check that $\alpha>0$; moreover, (2.11), (2.17), (2.18), and (2.13) yield

$$
|a(\hat{\mathbf{u}}, \hat{\mathbf{u}} ; \omega)| \geq \alpha C_{1}(\Omega)\|\hat{\mathbf{u}}(\omega)\|_{1, \Omega}^{2} .
$$

This and (2.14) lead to the following inequality:

$$
\|\hat{\mathbf{u}}(\omega)\|_{1, \Omega} \leq\left[1, \alpha C_{1}(\Omega)\right]^{-1}\|\hat{\mathbf{f}}(\omega)\|_{, \Omega} .
$$

If $\mathbf{f} \in L^{2}\left(\mathbb{R}, L^{2}(\Omega)\right)$, then $\hat{\mathbf{f}} \in L^{2}\left(\mathbb{R}, L^{2}(\Omega)\right)$ too, and $\|\hat{\mathbf{f}}\|_{, \Omega} \in L^{2}(\mathbb{R})$; therefore, by virtue of $(2.19)$, we have $\hat{\mathbf{u}} \in L^{2}\left(\mathbb{R}, H^{1}(\Omega)\right)$. Since the Fourier transform and its inverse are continuous mappings of $L^{2}$ onto itself, the thesis follows.

Finally, well-known results about elliptic problems imply that, if $G, \sigma$, and $\partial \Omega$ are sufficiently regular, the weak solution $\mathbf{u}$ of $(2.2)$ is indeed a classical solution, i.e., $\mathbf{u} \in$ $L^{2}\left(\mathbb{R}, H^{2}(\Omega)\right)$. Before stating the next theorem let us recall some properties of $\mathbb{G}$ :

Proposition 2.1. If $G$ satisfies i)-iii) then, for each fixed $\omega \in \mathbb{R}$, the bilinear form

$$
a_{1}(\mathbf{u}, \mathbf{v} ; \omega)=\int_{\Omega} \mathbb{G}(\mathbf{x}, \omega) \nabla \mathbf{u}(\mathbf{x}) \nabla \mathbf{v}^{*}(\mathbf{x}) d \mathbf{x}
$$

is strongly elliptic and its coefficients are uniformly Lipschitz. Moreover, there exist $\gamma, K>0$ such that

$$
\begin{aligned}
\left|a_{1}(\mathbf{u}, \mathbf{u} ; \omega)\right| \geq \gamma\|\nabla \mathbf{u}\|_{\Omega}^{2} \quad \forall \omega \in \mathbb{R}, \\
\left|\mathbb{G}_{i j k l}\left(\mathbf{x}_{1}, \omega\right)-\mathbb{G}_{i j k l}\left(\mathbf{x}_{2}, \omega\right)\right| \leq K\left|\mathbf{x}_{1}-\mathbf{x}_{2}\right| \quad \forall \mathbf{x}_{1}, \mathbf{x}_{2} \in \bar{\Omega} \quad \forall \omega \in \mathbb{R} .
\end{aligned}
$$

Proof. Inequality (2.21) follows immediately from (2.8), (2.15), and (2.16). In fact, we have $\gamma=\min \left\{\gamma_{1}, \gamma_{2}, \inf _{|\omega| \in I} \gamma(\omega)\right\}>0$. Besides, i $)_{2}$ implies that $\mathbb{G}(\cdot, \omega)$ is uniformly Lipschitz on $\bar{\Omega}$ for each fixed $\omega \in \mathbb{R}$, whereas i), implies that $\mathbb{G}(\mathbf{x}, \cdot)$ is absolutely continuous on $\mathbb{R}$ and $\lim _{\omega \rightarrow \infty} \mathbb{G}(\mathbf{x}, \omega)=G_{0}(\mathbf{x})$.

This is sufficient to ensure (2.22). 
THEOREM 2.3. If $G$ satisfies i)-iii), $\sigma \in C^{1}(\partial \Omega)$ and $\partial \Omega$ is of $C^{2}$-class then, for every $\mathbf{f} \in L^{2}\left(\mathbb{R}, L^{2}(\Omega)\right)$, the integro-differential problem (2.2) has one and only one solution $\mathbf{u} \in L^{2}\left(\mathbb{R}, H^{2}(\Omega)\right)$.

Proof. In fact (see [8], Theorem 2.4.2.6), if $G$ satisfies i)-iii), $\sigma \in C^{1}(\partial \Omega)$ and $\partial \Omega$ is of $C^{2}$-class and $\hat{\mathbf{f}}(\cdot, \omega) \in L^{2}(\Omega)$, then the solution $\hat{\mathbf{u}}(\cdot, \omega)$ of the elliptic problem (2.5) belongs to $H^{2}(\Omega)$. Moreover, the following a priori estimate holds for each $\omega \in \mathbb{R}$ :

$$
\|\hat{\mathbf{u}}(\omega)\|_{2, \Omega} \leq C^{\prime}(\omega)\left(\|\hat{\mathbf{f}}(\omega)\|_{, \Omega}+\|\hat{\mathbf{u}}(\omega)\|_{1, \Omega}\right) .
$$

$C^{\prime}$ depends on $\omega$ through the ellipticity constant of $a_{1}(\mathbf{u}, \mathbf{v}, \omega)$ and the Lipschitz constant of its coefficients; so $(2.20)$ and (2.21) ensure that $\sup _{\omega \in \mathbb{R}} C^{\prime}(\omega)=C^{\prime \prime}<\infty$. Hence it follows that

$$
\|\hat{\mathbf{u}}(\omega)\|_{2, \Omega} \leq C^{\prime \prime}\left(\|\hat{\mathbf{f}}(\omega)\|_{, \Omega}+\|\hat{\mathbf{u}}(\omega)\|_{1, \Omega}\right) .
$$

By the properties of the Fourier transform, $\mathbf{f} \in L^{2}\left(\mathbb{R}, L^{2}(\Omega)\right)$ and (2.19) imply $\mathbf{u} \in$ $L^{2}\left(\mathbb{R}, H^{2}(\Omega)\right)$.

3. Viscous boundary conditions. We now consider a viscoelastic body occupying a bounded domain $\Omega$ when a subset $\Gamma_{0}$ of its boundary $\partial \Omega$ is fixed and the remaining part $\Gamma_{1}=\partial \Omega \backslash \Gamma_{0}$ is subject to a viscous force. In this case quasi-static behaviour of the material is governed by the following system:

$$
\begin{cases}\nabla \cdot \mathbf{T}(\mathbf{x}, t)+\mathbf{f}(\mathbf{x}, t)=\mathbf{0} & (\mathbf{x}, t) \in \Omega \times \mathbb{R} \\ \mathbf{u}(\mathbf{x}, t)=\mathbf{0} & (\mathbf{x}, t) \in \Gamma_{0} \times \mathbb{R} \\ \lambda(\mathbf{x}) \dot{\mathbf{u}}(\mathbf{x}, t)+\mathbf{T}(\mathbf{x}, t) \cdot \mathbf{n}=\mathbf{0} & (\mathbf{x}, t) \in \Gamma_{1} \times \mathbb{R}\end{cases}
$$

where $\lambda(\mathbf{x})>0, \forall \mathbf{x} \in \bar{\Gamma}_{1}$ represents the viscosity coefficient corresponding to the dissipative stress on $\Gamma_{1}$. Substituting the constitutive equation (1.1) into (3.1) we obtain the following integro-differential problem:

$$
\begin{cases}\nabla \cdot\left(G_{0}(\mathbf{x}) \nabla \mathbf{u}(\mathbf{x}, t)+\int_{0}^{\infty} \dot{G}(\mathbf{x}, s) \nabla \mathbf{u}(\mathbf{x}, t-s) d s\right)+\mathbf{f}(\mathbf{x}, t)=\mathbf{0} & (\mathbf{x}, t) \in \Omega \times \mathbb{R} \\ \mathbf{u}(\mathbf{x}, t)=\mathbf{0} & (\mathbf{x}, t) \in \Gamma_{0} \times \mathbb{R} \\ \lambda(\mathbf{x}) \dot{\mathbf{u}}(\mathbf{x}, t)+G_{0}(\mathbf{x}) \nabla \mathbf{u}(\mathbf{x}, t) \cdot \mathbf{n}+\int_{0}^{\infty} \dot{G}(\mathbf{x}, s) \nabla \mathbf{u}(\mathbf{x}, t-s) \cdot \mathbf{n} d s=\mathbf{0} & (\mathbf{x}, t) \in \Gamma_{1} \times \mathbb{R}\end{cases}
$$

In treating such a kind of mixed boundary conditions (see [13]) we make use of the functional space $V=H_{0}^{1}\left(\Omega \cup \Gamma_{1}\right)$ which denotes the closure, in the $H^{1}$ norm, of $C^{\infty}(\Omega)$ functions whose support is a compact subset of $\Omega \cup \Gamma_{1}$.

Following Lions ([9]) we define a weak solution for problem (3.2) as follows.

DEFInition 3.1. A function $\mathbf{u}$ is said to be a weak solution of the quasi-static problem (3.2), with $\mathbf{f} \in L^{2}\left(\mathbb{R}, L^{2}(\Omega)\right)$, if $\mathbf{u} \in L^{2}(\mathbb{R}, V)$ and if, $\forall \mathbf{v} \in V$ and $\forall t \in \mathbb{R}$, it satisfies the following equality in variational form:

$$
\begin{aligned}
\int_{\Omega}\left(G_{0}(\mathbf{x}) \nabla \mathbf{u}(\mathbf{x}, t)+\int_{0}^{\infty} \dot{G}(\mathbf{x}, s)\right. & \nabla \mathbf{u}(\mathbf{x}, t-s) d s) \cdot \nabla \mathbf{v}(\mathbf{x}) d \mathbf{x} \\
& +\frac{d}{d t} \int_{\Gamma_{1}} \lambda(\mathbf{x}) \mathbf{u}(\mathbf{x}, t) \mathbf{v}(\mathbf{x}) d \mathbf{a}=\int_{\Omega} \mathbf{f}(\mathbf{x}, t) \mathbf{v}(\mathbf{x}) d \mathbf{x} .
\end{aligned}
$$


Then the Fourier transformed problem of (3.2) is defined for each $\omega \in \mathbb{R}$ as

$$
\begin{cases}\nabla \cdot(\mathbb{G}(\mathbf{x}, \omega) \nabla \hat{\mathbf{u}}(\mathbf{x}, \omega))+\hat{\mathbf{f}}(\mathbf{x}, \omega)=\mathbf{0} & \mathbf{x} \in \Omega, \\ \hat{\mathbf{u}}(\mathbf{x}, \omega)=\mathbf{0} & \mathbf{x} \in \Gamma_{0}, \\ i \omega \lambda(\mathbf{x}) \hat{\mathbf{u}}(\mathbf{x}, \omega)+(\mathbb{G}(\mathbf{x}, \omega) \nabla \hat{\mathbf{u}}(\mathbf{x}, \omega)) \cdot \mathbf{n}=\mathbf{0} & \mathbf{x} \in \Gamma_{1} .\end{cases}
$$

Definition 3.2. For each fixed $\omega \in \mathbb{R}$, $\hat{\mathbf{u}}$ is said to be a weak solution of problem (3.2) if $\hat{\mathbf{u}} \in V$ and if

$$
\int_{\Omega} \mathbb{G}(\mathbf{x}, \omega) \nabla \hat{\mathbf{u}}(\mathbf{x}) \nabla \mathbf{v}^{*}(\mathbf{x}) d \mathbf{x}+i \omega \int_{\Gamma_{1}} \lambda(\mathbf{x}) \hat{\mathbf{u}}(\mathbf{x}) \mathbf{v}^{*}(\mathbf{x}) d \mathbf{a}=\int_{\Omega} \hat{\mathbf{f}}(\mathbf{x}, \omega) \mathbf{v}^{*}(\mathbf{x}) d \mathbf{x}
$$

holds for every $\mathbf{v} \in V$.

Definitions 3.1 and 3.2 are strictly related like the corresponding definitions of the previous section, in the sense explained in Remark 2.1 .

We denote by meas $\left(\Gamma_{0}\right)$ the measure of $\Gamma_{0}$ in the $\partial \Omega$-measure and discuss separately the cases meas $\left(\Gamma_{0}\right)>0$ and meas $\left(\Gamma_{0}\right)=0$. We shall see that these cases are fairly different in that the first one is always well-posed if $\mathbf{f} \in L^{2}\left(\mathbb{R}, L^{2}(\Omega)\right)$, whereas the second needs further requirements in order to be solved.

Theorem 3.1. For each fixed $\omega \in \mathbb{R}$, if $G$ satisfies i)-iii) and if meas $\left(\Gamma_{0}\right) \neq 0$, then $\forall \hat{\mathbf{f}}(\cdot, \omega) \in L^{2}(\Omega)$, there exists one and only one weak solution $\hat{\mathbf{u}}(\cdot, \omega) \in V$ of $(3.4)$.

Proof. As in the previous section we show that the bilinear form

$$
a(\mathbf{u}, \mathbf{v} ; \omega)=\int_{\Omega} \mathbb{G}(\mathbf{x}, \omega) \nabla \mathbf{u}(\mathbf{x}) \nabla \mathbf{v}^{*}(\mathbf{x}) d \mathbf{x}+i \omega \int_{\Gamma_{1}} \lambda(\mathbf{x}) \mathbf{u}(\mathbf{x}) \mathbf{v}^{*}(\mathbf{x}) d \mathbf{a}
$$

is coercive in $V$, i.e., there exists $\beta(\omega)>0$ such that

$$
|a(\mathbf{u}, \mathbf{u} ; \omega)| \geq \beta(\omega)\|\mathbf{u}\|_{1, \Omega}^{2}
$$

For this purpose, let us remark that, if meas $\left(\Gamma_{0}\right) \neq 0$, Poincaré's inequality

$$
\|\nabla \mathbf{u}\|_{, \Omega}^{2} \geq C_{2}(\Omega)\|\mathbf{u}\|_{1, \Omega}^{2}
$$

holds for every $\mathbf{u} \in v$ (see [13], Lemma 1.46).

When $\omega \neq 0$, since $\lambda=\inf _{\mathbf{x} \in \Gamma_{1}} \lambda(\mathbf{x})>0$ and

$$
\operatorname{Im}\{a(\mathbf{u}, \mathbf{u} ; \omega)\}=-\int_{\Omega} \hat{\dot{G}}_{s}(\mathbf{x}, \omega) \nabla \mathbf{u}(\mathbf{x}) \nabla \mathbf{u}^{*}(\mathbf{x}) d \mathbf{x}+\omega \int_{\Gamma_{1}} \lambda(\mathbf{x}) \mathbf{u}(\mathbf{x}) \mathbf{u}^{*}(\mathbf{x}) d \mathbf{a}
$$

(2.8) and (3.6) yield

$$
|a(\mathbf{u}, \mathbf{u} ; \omega)| \geq|\operatorname{Im}\{a(\mathbf{u}, \mathbf{u} ; \omega)\}| \geq \gamma_{s}(\omega)\|\nabla \mathbf{u}\|_{, \Omega}^{2}+\lambda|\omega|\|\mathbf{u}\|_{, \partial \Omega}^{2} \geq \beta(\omega)\|\mathbf{u}\|_{1, \Omega}^{2}
$$

where $\beta(\omega)=C_{2}(\Omega) \gamma_{s}(\omega)$. On the other hand, when $\omega=0$, by $(1.4)_{2}$ we obtain

$$
|a(\mathbf{u}, \mathbf{u} ; 0)| \geq \operatorname{Re}\{a(\mathbf{u}, \mathbf{u} ; 0)\}=\int_{\Omega} G_{\infty}(\mathbf{x}) \nabla \mathbf{u}(\mathbf{x}) \nabla \mathbf{u}^{*}(\mathbf{x}) d \mathbf{x} \geq \gamma_{\infty}\|\nabla \mathbf{u}\|_{, \Omega}^{2} \geq \beta_{\infty}\|\mathbf{u}\|_{1, \Omega}^{2}
$$

Hence $a(\mathbf{u}, \mathbf{u} ; \omega)$ is coercive for each fixed $\omega \in \mathbb{R}$, and the thesis follows. 
THEOREM 3.2. Under the hypotheses of Theorem 3.1 on $G$ and $\Gamma_{0}$, for every $\mathbf{f} \in$ $L^{2}\left(\mathbb{R}, L^{2}(\Omega)\right)$, the integro-differential problem (3.2) has one and only one weak solution $\mathbf{u} \in L^{2}(\mathbb{R}, V)$.

Proof. By Theorem 3.1, for each $\omega$ such that $\hat{\mathbf{f}}(\cdot, \omega) \in L^{2}(\Omega)$, problem (3.4) admits one and only one weak solution $\hat{\mathbf{u}}(\cdot, \omega) \in V$ and we have

$$
|a(\hat{\mathbf{u}}, \hat{\mathbf{u}} ; \omega)|=\left|\int_{\Omega} \hat{\mathbf{f}}(\mathbf{x}, \omega) \hat{\mathbf{u}}^{*}(\mathbf{x}) d \mathbf{x}\right| \leq\|\hat{\mathbf{f}}\|_{, \Omega}\|\hat{\mathbf{u}}\|_{, \Omega} \leq\|\hat{\mathbf{f}}\|_{. \Omega}\|\hat{\mathbf{u}}\|_{1, \Omega} .
$$

Moreover, by virtue of (2.15), (2.16) and Poincaré's inequality (3.6), we obtain

$$
\begin{aligned}
& |a(\hat{\mathbf{u}}, \hat{\mathbf{u}} ; \omega)| \geq \operatorname{Re}\{a(\hat{\mathbf{u}}, \hat{\mathbf{u}} ; \omega)\} \geq \gamma_{1}\|\nabla \hat{\mathbf{u}}\|_{, \Omega}^{2} \geq \beta_{1}\|\hat{\mathbf{u}}\|_{1, \Omega}^{2} \quad \forall \omega \in\left[-\omega_{1}, \omega_{1}\right] \\
& |a(\hat{\mathbf{u}}, \hat{\mathbf{u}} ; \omega)| \geq \operatorname{Re}\{a(\hat{\mathbf{u}}, \hat{\mathbf{u}} ; \omega)\} \geq \gamma_{2}\|\nabla \hat{\mathbf{u}}\|_{, \Omega}^{2} \geq \beta_{2}\|\hat{\mathbf{u}}\|_{1, \Omega}^{2} \quad \forall|\omega| \geq \omega_{2} .
\end{aligned}
$$

If $I=\left[\omega_{1}, \omega_{2}\right]$ and $\beta=\min \left\{\beta_{1}, \beta_{2}, \inf _{|\omega| \in I} \beta(\omega)\right\}>0$, then $(3.7)$ and the latter inequalities lead to

$$
|a(\hat{\mathbf{u}}, \hat{\mathbf{u}} ; \omega)| \geq \beta\|\hat{\mathbf{u}}(\omega)\|_{1, \Omega}^{2}
$$

In this way, combining the last inequality with (3.8) we conclude that

$$
\|\hat{\mathbf{u}}(\omega)\|_{1, \Omega} \leq \beta^{-1}\|\hat{\mathbf{f}}(\omega)\|_{, \Omega}
$$

Since $\mathbf{f}, \hat{\mathbf{f}} \in L^{2}\left(\mathbb{R}, L^{2}(\Omega)\right),(3.9)$ ensures that $\hat{\mathbf{u}} \in L^{2}(\mathbb{R}, V)$.

The Fourier transform is an automorphism on $L^{2}$. Therefore, this is sufficient to prove the existence of one and only one weak solution $\mathbf{u} \in L^{2}(\mathbb{R}, V)$.

Finally, we consider problem $(3.2)$ when $\Gamma_{0}=\varnothing$. In this case $V=H^{1}(\Omega)$.

Observe that such a problem is not solvable in general; more precisely, its solution is not unique. (In fact, bounded functions of the type $\mathbf{u}(\mathbf{x}, t)=$ const. satisfy (3.2) with $\mathbf{f}=\mathbf{0}$ if $\Gamma_{0}=\varnothing$.) Nevertheless, we shall see that the homogeneous problem, associated to (3.2), only admits the trivial solution within the functional space $L^{2}\left(\mathbb{R}, H^{1}(\Omega)\right)$. Anyway, in order to ensure existence of solutions in such a functional space, it is no longer sufficient that $\mathbf{f} \in L^{2}\left(\mathbb{R}, L^{2}(\Omega)\right)$, but one must require also that the impulse

$$
\mathbf{g}(\mathbf{x}, t)=\int_{-\infty}^{t} \mathbf{f}(\mathbf{x}, s) d s
$$

belongs to the space $L^{2}\left(\mathbb{R}, L^{2}(\Omega)\right)$. The difficulties arising in $(3.2)$, when $\Gamma_{0}=\varnothing$ and $\Gamma_{1}=\partial \Omega$, are physically reasonable, because, by virtue of $(3.2)_{3}$, the material is neither fixed nor elastically attracted on the boundary, whereas the integro-differential equation $(3.2)_{1}$ involves at most the displacement gradient. In some problems of this kind (such as the similar one with Neumann boundary condition) the solution is unique only among the functions with vanishing spatial average, and its exists only if the supply $\mathbf{f}$ has the same feature; this is because, roughly speaking, the problem cannot control the "displacement on the whole" of the body.

On the contrary, the boundary viscosity $(3.2)_{3}$ controls, at least in part, the "motion on the whole" of the body because it depends on the displacement velocity. In this way one 
can find solutions to the problem (3.2) with nonvanishing spatial average. Unfortunately, the nature of the boundary force requires that the impulse $\mathbf{g}$, associated to $\mathbf{f}$ by $(3.10)$, belong to $H^{1}\left(\mathbb{R}, L^{2}(\Omega)\right)$, in order to obtain solutions in $L^{2}\left(\mathbb{R}, H^{1}(\Omega)\right)$.

Finally, observe that if $\mathbf{g} \in H^{1}\left(\mathbb{R}, L^{2}(\Omega)\right)$ then $\mathbf{f} \in L^{2}\left(\mathbb{R}, L^{2}(\Omega)\right)$ must have vanishing time-average on $\mathbb{R}$, but not every function $\mathbf{f} \in L^{2}\left(\mathbb{R}, L^{2}(\Omega)\right)$, with vanishing time-average on $\mathbb{R}$, is such that $\mathbf{g} \in H^{1}\left(\mathbb{R}, L^{2}(\Omega)\right)$ (consider, as an example, $f(t)=g^{\prime}(t)=-t\left(t^{2}+\right.$ $1)^{-5 / 4}$ and $\left.g(t)=2\left(t^{2}+1\right)^{-1 / 4}\right)$.

THEOREM 3.3. Under the hypotheses of Theorem 3.1 on $G$, for each $\mathbf{f}$ such that $\mathbf{g}$, defined by (3.10), belongs to $H^{1}\left(\mathbb{R}, L^{2}(\Omega)\right.$ ), the integro-differential problem (3.2) with $\Gamma_{0}=\varnothing$ has one and only one weak solution $\mathbf{u} \in L^{2}\left(\mathbb{R}, H^{1}(\Omega)\right)$.

Proof. First of all observe that, when $\Gamma_{0}=\varnothing$, Theorem 3.1 still holds for each $\omega \neq 0$. In fact, by (2.13), inequality (3.7) can be easily proved if we take $\beta(\omega)=$ $C_{1}(\Omega) \cdot \min \left(\gamma_{s}(\omega), \lambda|\omega|\right)$. In this way, the Fourier transform of the homogeneous problem associated to $(3.2)$ with $\Gamma_{0}=\varnothing$ only admits the trivial solution $\hat{\mathbf{u}}(\cdot, \omega)=0 \forall \omega \neq 0$. Since such a solution can be extended, by continuity, to $\omega=0$, and since the Fourier transform is injective, this implies that the solution to (3.2) with $\Gamma_{0}=\varnothing$, if it exists in $L^{2}\left(\mathbb{R}, H^{1}(\Omega)\right)$, is unique. Besides, by virtue of $(3.7),(2.15)$, and (2.16) we can find two positive constants $\omega_{1}$ and $\omega_{2}$ such that

$$
\begin{aligned}
2|a(\hat{\mathbf{u}}, \hat{\mathbf{u}} ; \omega)| & \geq|\operatorname{Re}\{a(\hat{\mathbf{u}}, \hat{\mathbf{u}} ; \omega)\}|+|\operatorname{Im}\{a(\hat{\mathbf{u}}, \hat{\mathbf{u}} ; \omega)\}| \\
& \left.\left.\geq \gamma_{1}\|\nabla \hat{\mathbf{u}}\|_{, \Omega}^{2}+\lambda|\omega|\|\hat{\mathbf{u}}\|_{, \partial \Omega}^{2} \geq \lambda C_{1}(\Omega)|\omega|\|\hat{\mathbf{u}}\|_{1, \Omega}^{2} \quad \forall|\omega| \in\right] 0, \omega_{1}\right], \\
2|a(\hat{\mathbf{u}}, \hat{\mathbf{u}} ; \omega)| & \geq|\operatorname{Re}\{a(\hat{\mathbf{u}}, \hat{\mathbf{u}} ; \omega)\}|+|\operatorname{Im}\{a(\hat{\mathbf{u}}, \hat{\mathbf{u}} ; \omega)\}| \\
& \geq \gamma_{2}\|\nabla \hat{\mathbf{u}}\|_{, \Omega}^{2}+\lambda|\omega|\|\hat{\mathbf{u}}\|_{, \partial \Omega}^{2} \geq \gamma_{2} C_{1}(\Omega)\|\hat{\mathbf{u}}\|_{1, \Omega}^{2} \quad \forall|\omega| \geq \omega_{2} .
\end{aligned}
$$

Remarking that $\beta(\omega)>0$ for $|\omega| \in\left[\omega_{1}, \omega_{2}\right]$, from (3.8) one gets

$$
\begin{array}{rr}
\|\hat{\mathbf{u}}(\omega)\|_{1, \Omega} \leq C_{3}(\Omega)\|\hat{\mathbf{f}}(\omega)\|_{, \Omega} & \forall|\omega| \geq \omega_{1} \\
\|\hat{\mathbf{u}}(\Omega)\|_{1, \Omega} \leq 2\left[\lambda C_{1}(\Omega)|\omega|\right]^{-1}\|\hat{\mathbf{f}}(\omega)\|_{, \Omega} & \left.\forall|\omega| \in] 0, \omega_{1}\right] .
\end{array}
$$

If $\mathbf{f}$ has a primitive in the sense of distributions and $\mathbf{g} \in H^{1}\left(\mathbb{R}, L^{2}(\Omega)\right)$ is defined by (3.10) then $\|\hat{\mathbf{f}}(\omega)\|_{, \Omega}=|\omega|\|\hat{\mathbf{g}}(\omega)\|_{, \Omega}$ so that from (3.14) it follows that

$$
\left.\left.\|\hat{\mathbf{u}}(\omega)\|_{1, \Omega} \leq C_{4}\|\hat{\mathbf{g}}(\omega)\|_{, \Omega} \quad \forall|\omega| \in\right] 0, \omega_{1}\right]
$$

Estimates (3.14) and (3.15) ensure that, if $\hat{\mathbf{g}}, \hat{\mathbf{f}} \in L^{2}\left(\mathbb{R}, L^{2}(\Omega)\right)$, then there exists a function $\hat{\mathbf{u}} \in L^{2}\left(\mathbb{R}, H^{1}(\Omega)\right)$ such that $\hat{\mathbf{u}}(\cdot, \omega)$ is a solution of (3.4) for each $\omega$ for which $\hat{\mathbf{f}}(\cdot, \omega) \in L^{2}(\Omega)$. By virtue of the Fourier transform and of its inverse, the integrodifferential problem (3.2) has one and only one solution $\mathbf{u} \in L^{2}\left(\mathbb{R}, H^{1}(\Omega)\right)$ whose Fourier transform is $\hat{\mathbf{u}}$.

Finally, if $G, \lambda$, and $\partial G$ are sufficiently regular, we can state that the weak solution $\mathbf{u}$ of $(2.2)$ with $\Gamma_{0}=\varnothing$ is indeed a classical solution, i.e., $\mathbf{u} \in L^{2}\left(\mathbb{R}, H^{2}(\Omega)\right)$. 
THEOREM 3.4. If $G$ satisfies i)-iii), $\lambda \in C^{1}(\partial \Omega)$, and $\partial \Omega$ is of $C^{2}$-class, then for every $\mathbf{f}$ such that $\mathbf{g}$, defined by (3.10), belongs to $H^{1}\left(\mathbb{R}, L^{2}(\Omega)\right)$, the integro-differential problem (3.2) with $\Gamma_{0}=\varnothing$ has one and only one solution $\mathbf{u} \in L^{2}\left(\mathbb{R}, H^{2}(\Omega)\right)$.

Proof. By using the same argument as in Theorem 2.3 we can say that, if $G$ satisfies i)-iii), $\lambda \in C^{1}(\partial \Omega), \partial \Omega$ is of $C^{2}$-class, and $\hat{\mathbf{f}}(\cdot, \omega) \in L^{2}(\Omega)$, then the solution $\hat{\mathbf{u}}(\cdot, \omega)$ of the elliptic problem (3.4), with $\Gamma_{0}=\varnothing$, if it exists, belongs to $H^{2}(\Omega)$ and satisfies inequality (2.24). Theorem 3.3 and (2.24) imply that $\hat{\mathbf{u}} \in L^{2}\left(\mathbb{R}, H^{2}(\Omega)\right)$; by properties of the Fourier transform the thesis follows.

Acknowledgment. This work has been performed under the auspices of C.N.R.G.N.F.M. and M.U.R.S.T., $60 \%$ and $40 \%$ respectively.

\section{REFERENCES}

[1] W. A. Day, The thermodynamics of simple materials with fading memory, Springer Tracts in Natural Philosophy, vol. 22, Springer-Verlag, New York, 1972

[2] M. Fabrizio, An existence and uniqueness theorem in quasi-static viscoelasticity, Quart. Appl. Math. 47, 1-8 (1989)

[3] M. Fabrizio and A. Morro, Viscoelastic relaxation function compatible with thermodynamics, J. Elasticity 19, 63-75 (1988)

[4] M. Fabrizio and A. Morro, On uniqueness in linear viscoelasticity: a family of counterexamples, Quart. Appl. Math. 45, 321-325 (1987)

[5] M. Fabrizio and A. Morro, Mathematical Problems in Linear Viscoelasticity, SIAM Studies in Applied Mathematics, vol. 12, Philadelphia, 1992

[6] G. Gentili, Alcune proprietà per la funzione di rilassamento in viscoelasticità lineare, Riv. Mat. Univ. Parma (4) 14, 121-133 (1988)

[7] D. Graffi, Sui problemi della ereditarietà lineare, Nuovo Cimento 5, 53-71 (1928)

[8] P. Grisvard, Elliptic Problems in Nonsmooth Domains, Pitman, London, 1985

[9] J. L. Lions, Equations Differentielles Operatiorınelles et Problèmes aux Limites, Springer-Verlag, Berlin-Gottingen-Heidelberg, 1961

[10] A. C. Pipkin, Lectures on Viscoelasticity Theory, Springer, Berlin, 1972

[11] R. E. Showalter, Hilbert Space Methods for Partial Differential Equations, Pitman, London and San Francisco, 1977

[12] F. Treves, Basic Linear Partial Differential Equations, Academic Press, New York, 1975

[13] G. M. Troianiello, Elliptic Differential Equations and Obstacle Problems, Plenum Press, New York, London, 1987 